\title{
ECOLOGIA DE XYLOCOPA (NEOXYLOCOPA) CEARENSIS (HYMENOPTERA, ANTHOPHORIDAE) NAS DUNAS LITORÂNEAS DE ABAETÉ, SALVADOR, BAHIA
}

\author{
Blandina F. Viana ${ }^{1,3}$ \\ Astrid M. P. Kleinert ${ }^{2}$ \\ Fabiana O. Silva ${ }^{1}$
}

\begin{abstract}
ECOLOGY OF XYLOCOPA (NEOXYLOCOPA) CEARENSIS (HYMENOPTERA, ANTHOPHORIDAE) IN ABAETÉ SAND DUNES, SALVADOR, BAHIA. The study was carried out in a 8.2 ha area in an environmental protection area of tropical sea coastal sand dune with "restinga" vegetation in Salvador, Bahia, northeastern Brazil. A total of 1760 bees of Xylocopa (Neoxylocopa) cearensis Ducke, 1910 were netted during the whole year on flowers of 43 plant species belonging to 26 botanic families. The majority of the individuals (79\%) concentrated their foraging activity in five plant species. Individuals foraged all day long being the greatest activity between $8 \mathrm{~h}$ and $14 \mathrm{~h}$. Similar proportions of young and old bees were sampled over the year. The density of substrates used for nesting was $4.56 /$ ha. In total, $94 \%$ of the nests were found in branches of Agaristha revoluta (Spr.) DC. (Ericaceae). The great occurrence $(68.7 \%)$ of old perforations indicates that the nests were used twice or more times by bees.
\end{abstract}

KEYWORDS. Xylocopa, floral resources, nests, dune.

\section{INTRODUÇÃO}

O gênero Xylocopa Latreille, 1802 ocorre principalmente nas regiões tropicais e subtropicais e menos de $10 \%$ das espécies são encontradas em zonas temperadas (GERLING et al., 1989). Das 750 espécies do gênero, 50 são conhecidas no Brasil (HuRD, 1978). Em geral, as espécies deste gênero apresentam hábito solitário, caracterizado pela independência das fêmeas na construção e aprovisionamento de seus ninhos e pela ausência de trabalho cooperativo, embora as fêmeas permaneçam no ninho após a emergência da cria. Embora os ninhos sejam fundados solitariamente, diferentes

1. Depto de Zoologia, Instituto de Biologia, Universidade Federal da Bahia, Rua Barão de Geremoabo s/ $\mathrm{n}^{\circ}$, Campus Universitário de Ondina, 40170-110, Salvador, BA, Brasil. (labea@ufba.br)

2. Depto de Ecologia, Instituto de Biociências, Universidade de São Paulo, Rua do Matão, travessa $14, n^{\circ} 321$, Cidade Universitária, Butantã, 05508-900, São Paulo, SP, Brasil. (astridkl@usp.br)

3. Pesquisador do CNPq. 
comportamentos de socialidade têm sido registrados em algumas espécies de Xylocopa, após a fundação dos ninhos (Gerling \& Hermann, 1978; Camillo \& Garófalo, 1982, 1989; CAMILlo et al., 1986; Gerling et al., 1989). A maioria das Xylocopa possui atributos como longevidade prolongada das fêmeas, sobreposição de gerações e tolerância à presença de co-específicos no ninho, considerados como requisitos para a evolução da eusocialidade (MiCHENER, 1974).

Em geral, as espécies subtropicais e tropicais apresentam bivoltismo (duas gerações, produzidas por uma mesma fêmea, por ano). Algumas apresentam multivoltismo, podendo ter de quatro a cinco gerações por ano. Nesses casos, os ninhos são freqüentemente reutilizados (SAKagami \& Laroca, 1971; Camillo \& Garófalo, 1989). De modo geral, as espécies constróem seus ninhos em material vegetal seco, morto ou apodrecido, perfurando-os ou utilizando cavidades pré-existentes, podendo ser lineares ou ramificados (Hurd \& Moure, 1963; Sage, 1968; Anzemberger, 1977; Camillo \& Garófalo, 1982; CAmillo et al., 1986).

Essas abelhas utilizam néctar e pólen como únicas fontes de alimento. Por apresentarem vida longa e ampla distribuição geográfica (HURD \& MOURE, 1963) a maioria das Xylocopa é generalista, visita grande número de espécies vegetais, concentrando-se porém em alguns recursos mais produtivos. Apresentam constância floral associada com a abundância de certas espécies vegetais em uma dada hora do dia, época do ano ou local (GerLING et al., 1989). A maioria das espécies de Xylocopa é diurna, visita diferentes flores em diferentes horas. Em alguns casos, a visita coincide com o horário da abertura da flor (CORBET \& WILLMER, 1980). Não costumam visitar uma mesma flor imediatamente após uma visita prévia, tornando-se assim eficazes agentes polinizadores. Essa característica está associada à marcação das flores por feromônios (FrankIE \& VINSON, 1977). A coleta de néctar é normalmente realizada de modo "legítimo", em nectários florais e/ou extraflorais. Em flores com néctar inacessível, podem também praticar o roubo, através de perfurações no cálice e na corola, utilizando suas gáleas maxilares. O pólen é coletado ativamente nas anteras, por varredura ou pela vibração nas anteras com deiscência poricida, ou passivamente, por ocasião da coleta de néctar (BUCHMANN, 1983).

São abundantes nos ambientes abertos de dunas e praias do nordeste do Brasil, caracterizados por umidade relativa e temperatura constantemente altas e por fortes ventos (acima de 4m/s). Xylocopa (Neoxylocopa) carbonaria Smith, 1854 foi a espécie mais abundante nas dunas do Maranhão (GoTTSBERGER et al., 1988).

São apresentados alguns aspectos da ecologia de $X$. cearensis relacionados à abundância sazonal, às plantas visitadas, ao modo de utilização dos recursos florais, aos substratos de nidificação, à densidade e distribuição espacial dos ninhos em um ambiente de restinga.

\section{MATERIAL E MÉTODOS}

O estudo foi desenvolvido em um sítio de 8,2 ha da Área de Proteção Ambiental das Lagoas e Dunas de Abaeté, Salvador, Bahia (12²56'S; 38 $21^{\prime}$ 'W) entre janeiro e dezembro de 1996, em intervalos de aproximadamente 10 dias. Realizou-se a amostragem das abelhas nas flores pelo método de varredura

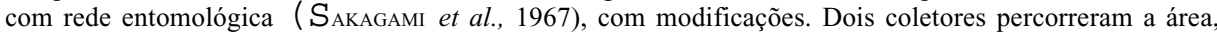
seguindo a mancha de vegetação em direções opostas. Para calcular a abundância relativa das abelhas nas espécies vegetais predominantemente visitadas, utilizou-se o método de probabilidade de Kato et al., 1952 apud LAROCA (1995). As abelhas encontram-se depositadas na coleção do Laboratório de Biologia e Ecologia de Abelhas (LABEA), Instituto de Biologia, Universidade Federal da Bahia, Salvador, BA. 
Avaliou-se a composição etária da população de $X$. cearensis ao longo do ano, de acordo com o padrão de desgaste alar (RebÊLo \& GARóFalo, 1991), em quatro grupos: 1, sem desgaste alar; 2, pequeno desgaste alar; 3, moderado desgaste alar; 4, grande desgaste alar.

A intensidade da floração das espécies vegetais foi monitorada mensalmente; para as espécies com flores isoladas, avaliou-se a produção de flores quantificando-se o número médio de flores e multiplicando-se este número pelo número total de indivíduos. Nas espécies com flores reunidas em inflorescências, o número médio de flores por inflorescência foi multiplicado pelo número total de inflorescências e este valor multiplicado pelo número total de indivíduos. A caracterização das flores incluiu a observação do horário de antese, tipo de recurso, cor, forma, tamanho (profundidade e diâmetro), sexualidade e simetria.

Utilizou-se o método “T-Square distance sampling” para determinar o padrão de distribuição espacial dos sítios nidificados em 30 pontos amostrais selecionados aleatoriamente na área. Cada ponto amostral originou quatro quadrantes, os quais eram percorridos, medindo-se a distância até o sítio nidificado mais próximo (x) e deste até o seu vizinho mais próximo (y). Utilizou-se o programa BASIC.TSQUARE.BAS (LuDWIG \& REYNOLDS, 1988) para a obtenção do índice de distribuição espacial (C).

Determinou-se a densidade dos ninhos e das espécies vegetais arbustivas pelo método dos quadrantes centrados, medindo-se as distâncias do ponto amostral até o indivíduo mais próximo (x) em cada quadrante, obtendo-se a estimativa da densidade pelo inverso do quadrado da densidade (GREIG-SMITH, 1983). A densidade das ervas e subarbustos foi avaliada qualitativamente. As exsicatas encontram-se no Herbário da Universidade Estadual de Feira de Santana (HUFS).

Para a identificação do tipo de substrato e o seu modo de utilização, registrou-se as espécies vegetais com ninhos, o número de orifícios existentes no galho e em toda a planta, e se os mesmos tinham sido escavados recentemente ou eram antigos. Medidas, em centímetros, foram tomadas dos diâmetros dos galhos e dos diâmetros internos de cada orifício.

\section{RESULTADOS}

Abundância diária e sazonal. X. cearensis predominou entre os Apoidea coletados nas flores, representando $42,7 \%$ dos indivíduos coletados. Os indivíduos visitaram flores durante todo o dia com maior freqüência entre 8 e $14 \mathrm{~h}$. O menor número de indivíduos foi coletado entre $16 \mathrm{e} 18 \mathrm{~h}$ (fig. 1). Estiveram ativos durante os 12 meses de amostragem (fig. 2).

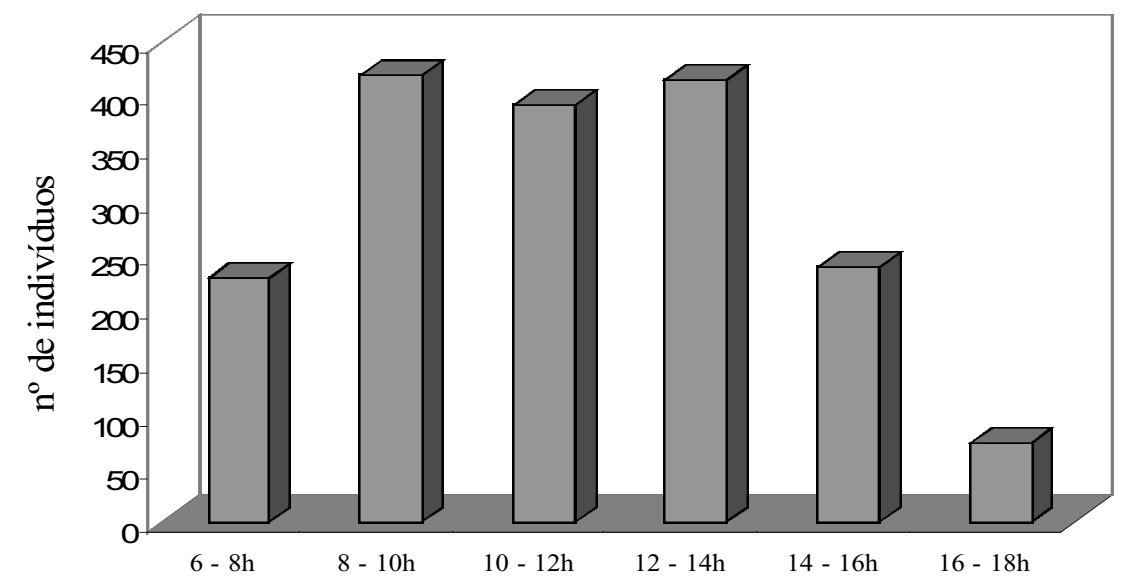

Fig. 1. Número total de indivíduos de Xylocopa (Neoxylocopa) cearensis coletados em cada intervalo de duas horas nas dunas litorâneas de Abaeté, Salvador, Bahia. 
Tanto machos quanto fêmeas foram mais abundantes nos meses menos chuvosos: janeiro a março e outubro a dezembro/1996 (fig.3), período que coincide também com a maior disponibilidade de recursos florais no campo (fig.4).

Composição etária. Os indivíduos distribuíram-se na população, em relação ao padrão de desgaste alar, em proporções relativamente similares. As maiores variações ocorreram entre os indivíduos mais jovens (idade 1, 164 indivíduos) que foram menos abundantes e os mais velhos (idade 4, 516 indivíduos), mais abundantes. Com as idades 2 e 3 detectaram-se 428 e 484 indivíduos, respectivamente. Em todos os meses foram coletados indivíduos de todas categorias etárias (fig. 5).

Distribuição dos ninhos e tipo de substrato. Os 107 sítios nidificados por $X$. cearensis, encontrados nas dunas de Abaeté apresentaram padrão de distribuição agregado $(\mathrm{C}=0,77)$. $\mathrm{O}$ valor médio das distâncias do ponto amostral até o primeiro sítio nidificado (média $=46,81 \mathrm{~m}$ ) foi quase o dobro deste até o seu vizinho mais próximo (média $=24,96 \mathrm{~m}$ ), indicando maior probabilidade de se encontrar um novo ninho próximo a outro previamente detectado. Foram encontrados 4,56 ninhos por ha, com distância média de 46,81 m entre os sítios nidificados. A maior parte dos ninhos (94\%) foi encontrada em Agaristha revoluta, Ericaceae, tanto em galhos secos caídos sobre o solo quanto no tronco da planta viva. $O$ diâmetro do substrato utilizado variou entre 4 e $17 \mathrm{~cm}$ contendo, na maioria, 1 a 2 ninhos (58\%) (figs. 6,7). O orifício de entrada circular localizado na superfície lateral dos galhos, com 10,35 $\mathrm{mm} \pm 1,79$ de diâmetro. Cerca de $68,7 \%$ eram antigos, indicando a possibilidade de terem sido reutilizados.

Utilização de recursos florais. Xylocopa cearensis visitou 43 espécies vegetais (tab. I), concentrando as visitas (79\% do total de indivíduos) em apenas 5 delas: Cuphea brachiata (27\%), Waltheria cinerescens (26\%), Acosmium bijugum (10\%), Humiria balsamifera (10\%) e Chamaecrista ramosa (6\%). Outras espécies visitadas somaram $21 \%$. Em janeiro, as abelhas concentravam-se em A. bijugum, embora tenham também visitado $C$. brachiata e $W$. cinerescens. No mês de fevereiro, exploraram mais intensamente $H$. balsamifera e A. bijugum (fig. 8). De março a maio, em C. brachiata, e entre junho a novembro, em $W$. cinerescens. Em dezembro, foram coletadas em proporções mais ou menos similares nas fontes florais preferenciais disponíveis, embora um maior número de indivíduos tenha visitado C. brachiata.

Durante todo o dia havia recursos florais disponíveis (fig. 9). Chamaecrista ramosa, a principal fonte de pólen, foi visitada predominantemente entre 6 e $8 \mathrm{~h}$, decrescendo o número de visitantes até às $12 \mathrm{~h}$, quando a quase totalidade de flores fechava. As duas principais fontes de néctar foram visitadas em horários diferentes. Em C. brachiata, o maior número de indivíduos foi coletado após as $12 \mathrm{~h} ; \mathrm{em} W$. cinerescens a maior freqüência ocorreu no período da manhã.

As flores das espécies preferencialmente visitadas por $X$. cearensis são todas melitófilas e hermafroditas (tab. II). Waltheria cinerescens é a espécie vegetal mais comum entre os arbustos e árvores na área de estudo. Humiria balsamifera e A. bijugum, embora tenham sido pouco representadas na área, apresentam alta intensidade floral. As densidades das ervas e subarbustos não foram avaliadas quantitativamente. Foi observado que $C$. brachiata e C. ramosa eram amplamente distribuídas nas dunas, formando largas manchas sobre o substrato de areia. 
$300-$
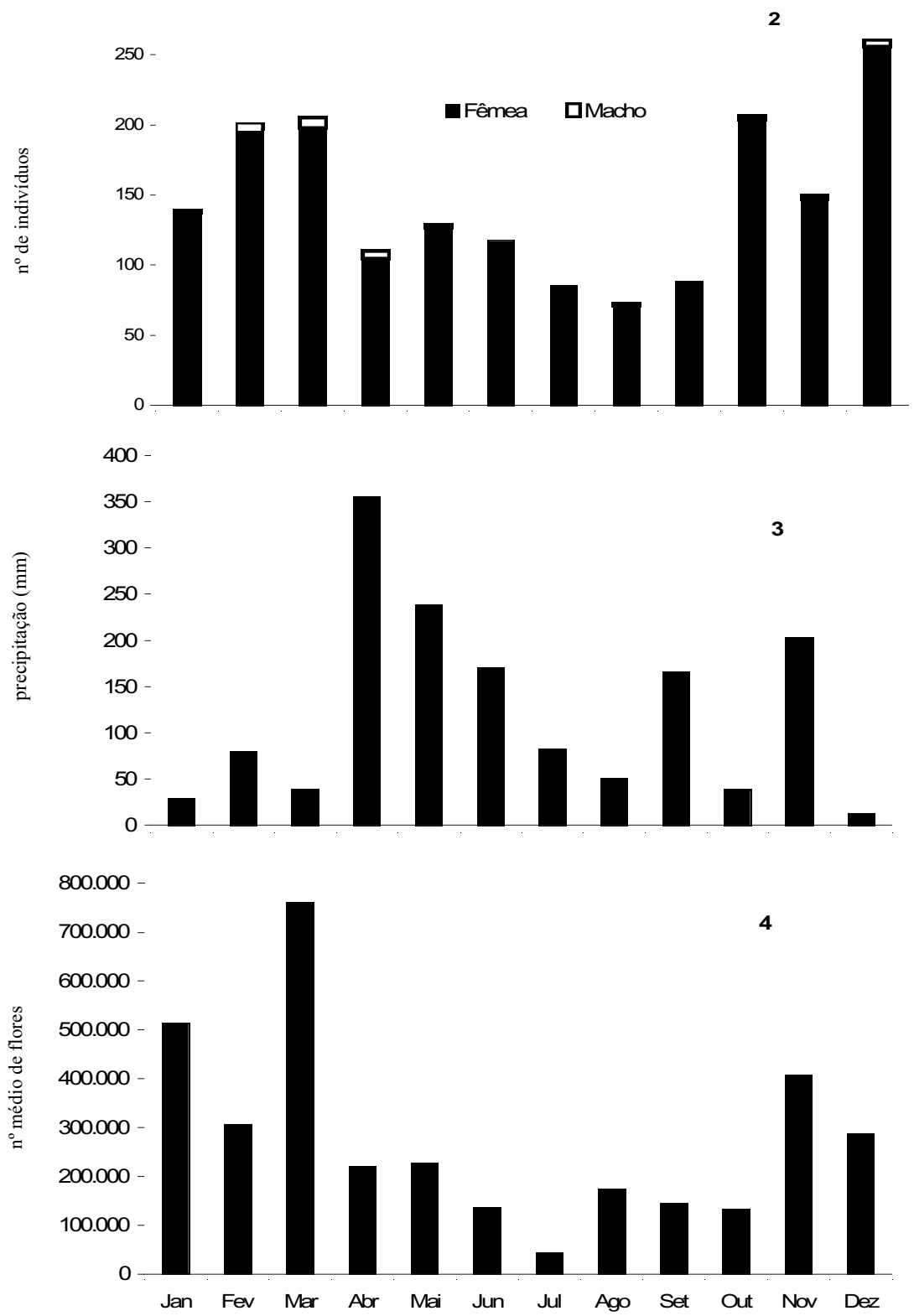

Figs. 2-4. Xylocopa (Neoxylocopa) cearensis, indivíduos coletados nas flores, nas dunas da Abaeté, Salvador, BA, no período de janeiro a dezembro de 1996: 2, número total de machos e fêmeas; 3, em relação à precipitação; 4 , em relação à intensidade de floração.

Iheringia, Sér. Zool., Porto Alegre, 92(4):47-57, 30 de dezembro 2002 


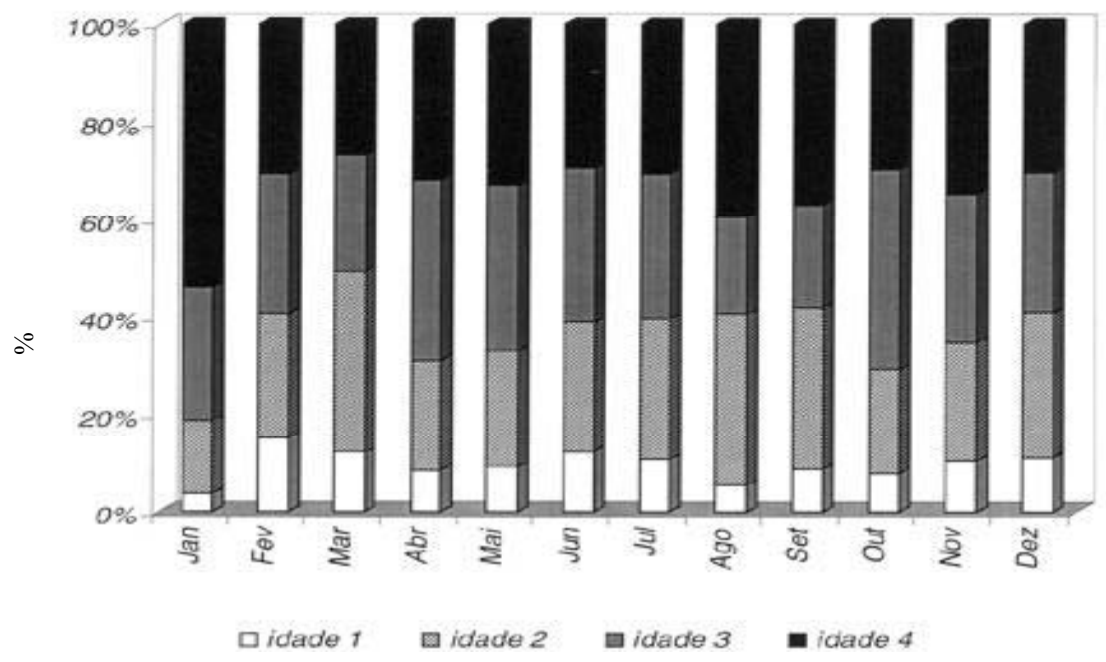

Fig. 5. Freqüência (\%) de indivíduos de Xylocopa (Neoxylocopa) cearensis, de acordo com a idade, ao longo dos meses, nas dunas litorâneas de Abaeté, Salvador, BA.
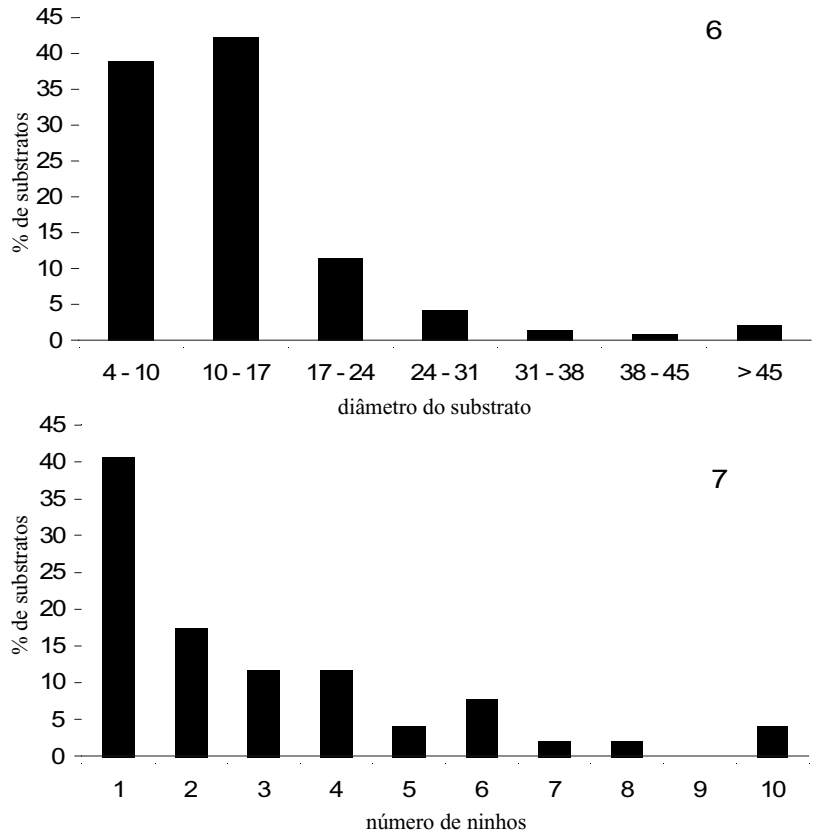

Fig. 6-7. Ninhos de Xylocopa (Neoxylocopa) cearensis amostrados no período de outubro de 1996 a maio de 1997 nas dunas de Abaeté, Salvador, BA: 6, diâmetros dos substratos com ninhos; 7, número de ninhos por substrato.

Iheringia, Sér. Zool., Porto Alegre, 92(4):47-57, 30 de dezembro 2002 


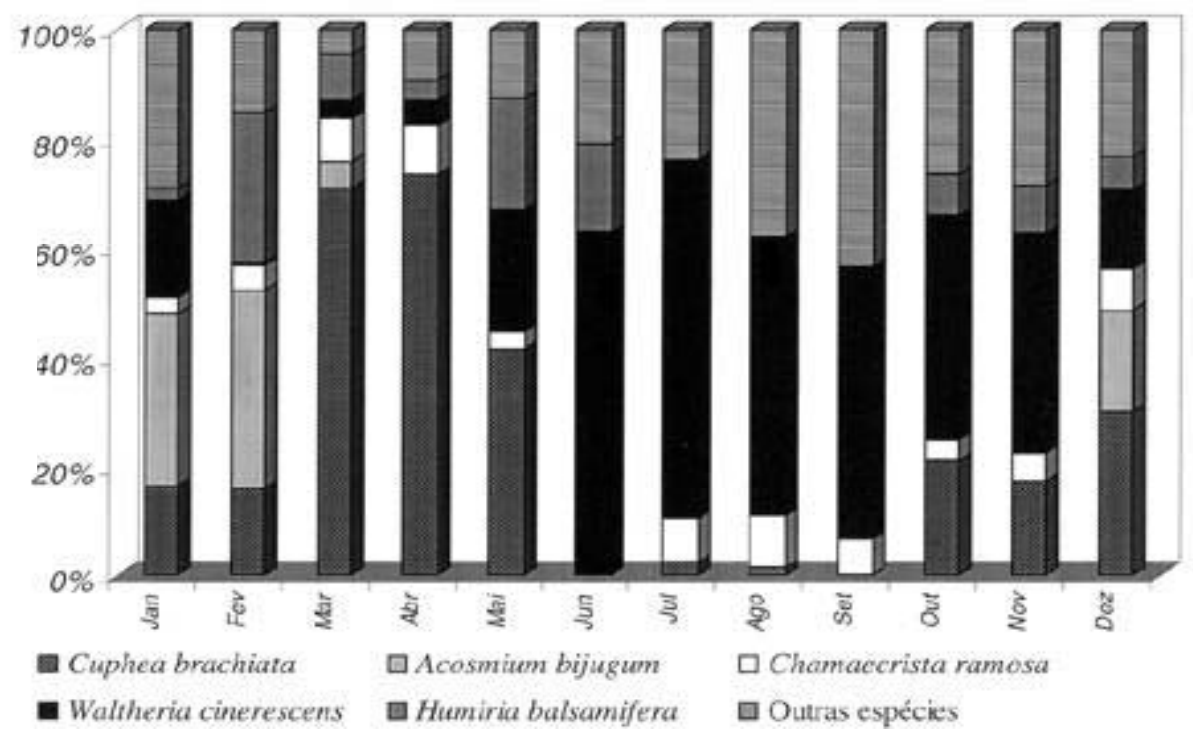

Fig. 8. Variação mensal na porcentagem de indivíduos de Xylocopa (Neoxylocopa) cearensis coletados nas espécies vegetais predominantemente visitadas, nas dunas litorâneas de Abaeté, Salvador, Bahia.

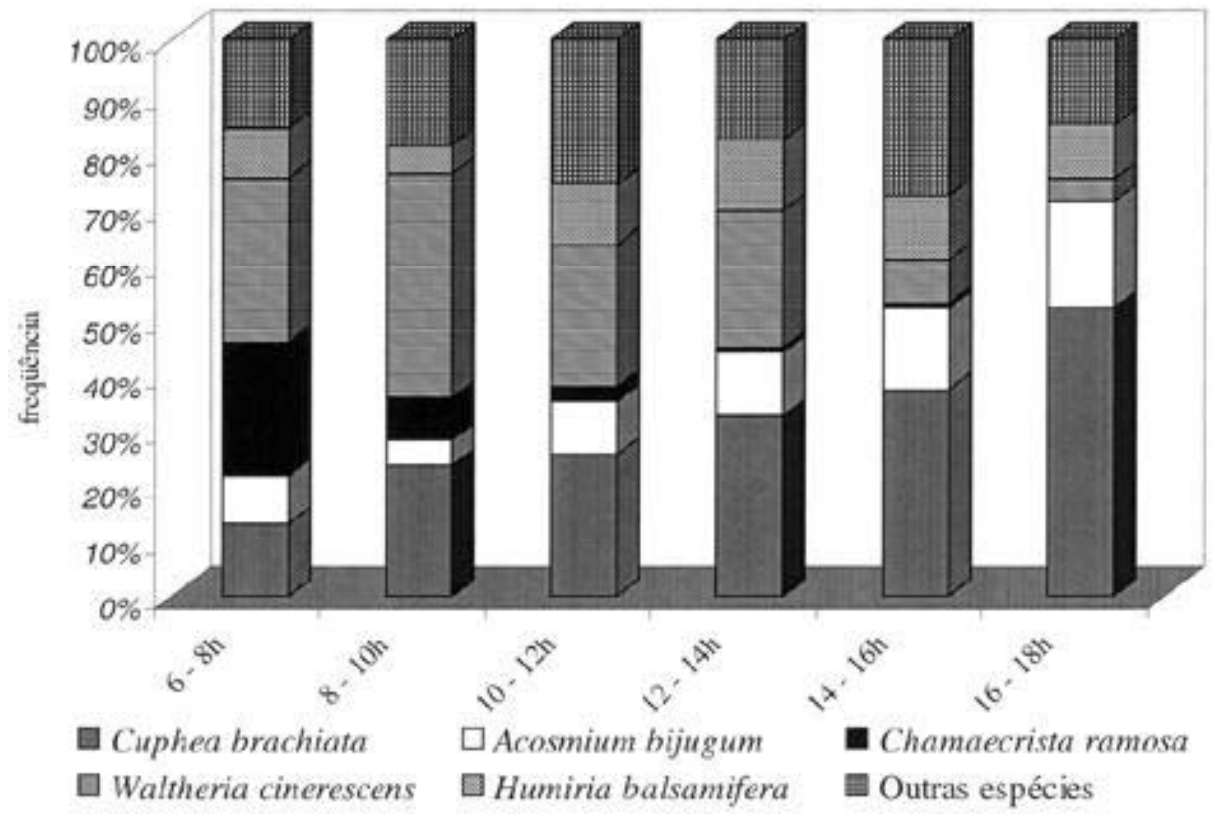

Fig. 9. Variação diária na freqüência (\%) de indivíduos de Xylocopa (Neoxylocopa) cearensis coletados nas espécies vegetais predominantemente visitadas nas dunas litorâneas de Abaeté. 
Tabela I. Número de machos e fêmeas de Xylocopa (Neoxylocopa) cearensis coletado em cada espécie vegetal, entre janeiro e dezembro/1996, nas dunas litorâneas de Abaeté, Salvador, Bahia.

\begin{tabular}{|c|c|c|c|c|}
\hline Família & Espécie & Machos & Fêmeas & Total \\
\hline ANACARDIACEAE & Tapirira guianensis Aubl. & & 20 & 20 \\
\hline \multirow[t]{5}{*}{ ASTERACEAE } & Conocliniopsis prasiifolia & & & \\
\hline & (DC.) R.M. King \& H. Rob. & & 8 & 8 \\
\hline & Mikania nitida (DC.) R.M. King \& H. Rob. & 1 & 12 & 13 \\
\hline & Stilpnopappus scaposus DC. & & 1 & 1 \\
\hline & Vernonia arenaria Mart. ex. DC. & & 43 & 43 \\
\hline BIGNONIACEAE & Tabebuia elliptica (A. DC.) Sandwith & & 2 & 2 \\
\hline \multirow[t]{2}{*}{ BURSERACEAE } & Protium bahianum Daly & & 7 & 7 \\
\hline & P. icicariba (DC.) Marchand & & 22 & 22 \\
\hline \multirow[t]{4}{*}{ CAESALPINIACEAE } & Chamaecrista ramosa var. ramosa & & & \\
\hline & (Vogel) H.S. Irwin \& Barneby & & 101 & 101 \\
\hline & Moldenhawera nutans L.P. Queiroz, & & & \\
\hline & G.P. Lewis \& Allkin & & 1 & 1 \\
\hline CAPPARACEAE & Dactylaena microphylla Eichler & 1 & 1 & 2 \\
\hline CLUSIACEAE & Kielmeyera argentea Choisy & & 2 & 2 \\
\hline CONVOLVULACEAE & Evolvulus sp. & & 1 & 1 \\
\hline ERICACEAE & Agaristha revoluta (Spr.) DC. & & 1 & 1 \\
\hline \multirow[t]{7}{*}{ FABACEAE } & Acosmium bijugum (Vogel) Yakovlev & 7 & 168 & 175 \\
\hline & Centrosema brasilianum (L.) Benth. & & 1 & 1 \\
\hline & C. coriaceum Benth. & & 2 & 2 \\
\hline & Stylosanthes viscosa (L.) Sw. & & 8 & 8 \\
\hline & Swartzia apetala var. subcordata R.S. Cowan & & 1 & 1 \\
\hline & Vigna halophila (Piper) Maréchal, & & & \\
\hline & Mascherpa \& Stainier & & 32 & 32 \\
\hline \multirow[t]{2}{*}{ HUMIRIACEAE } & Humiria balsamifera var. parvifolia & & & \\
\hline & (A. Juss.) Cuatrec. & 8 & 161 & 169 \\
\hline ICACINACEAE & Emmotum affine Miers & & 1 & 1 \\
\hline LAMIACEAE & Eriope blanchetii (Benth.) R. M. Harley & & 56 & 56 \\
\hline \multirow[t]{2}{*}{ LORANTHACEAE } & Struthanthus flexilis (Rusby) Kuijt & & 2 & 2 \\
\hline & Martius ex G. Don & & 9 & 9 \\
\hline LYTHRACEAE & Cuphea brachiata (Mart.) Koehne & 1 & 490 & 491 \\
\hline \multirow{2}{*}{ MELASTOMATACEAE } & Comolia ovalifolia (DC.) Triana & & 13 & 13 \\
\hline & Pterolepis edmundoi Brade \& Markgraf. & & 4 & 4 \\
\hline MYRTACEAE & Myrcia sp. & & 3 & 3 \\
\hline PASSIFLORACEAE & Passiflora edulis Sims & & 2 & 2 \\
\hline \multirow{4}{*}{ POLYGALACEAE } & Polygala psamophila Chod. \& Hub. & 1 & 1 & 2 \\
\hline & Polygala sp. & & 1 & 1 \\
\hline & Coccoloba cordifolia Meisn. & 1 & 27 & 28 \\
\hline & C. ramosissima Wedd. & & 6 & 6 \\
\hline \multirow[t]{3}{*}{ RUBIACEAE } & Borreria cymosa (Spreng.) Cham. \& Schltdl. & & 11 & 11 \\
\hline & B. verticillata (L.) G. Mey. & & 2 & 2 \\
\hline & Borreria sp. & & 5 & 5 \\
\hline SAPINDACEAE & Cardiospermum integerrimum Radlk. & & 3 & 3 \\
\hline SAPOTACEAE & Manilkara salzmannii (A. DC.) H.J. Lam & & 29 & 29 \\
\hline STERCULIACEAE & Waltheria cinerescens St. Hilaire & 8 & 452 & 460 \\
\hline VELLOZIACEAE & Vellozia dasypus Seub. & & 4 & 4 \\
\hline \multirow[t]{2}{*}{ VERBENACEAE } & Lantana camara $\mathrm{L}$. & & 5 & 5 \\
\hline & Vitex cymosa Bertero ex Spreng. & & 11 & 11 \\
\hline TOTAL & & 28 & 1732 & 1760 \\
\hline
\end{tabular}

Iheringia, Sér. Zool., Porto Alegre, 92(4):47-57, 30 de dezembro 2002 
Tabela II. Características das espécies vegetais melitófilas predominantemente visitadas por Xylocopa (Neoxylocopa) cearensis em Abaeté, Salvador, Bahia $\left({ }^{1}\right.$, a densidade das ervas e subarbustos não foi avaliada quantitativamente; A, amarela; Ar, arbusto; C, creme; Co, cornucópia; H, hermafrodita; N, néctar; P, pólen; R, radial; Sar, subarbusto; St, "steady-state"; Z, zigomorfa).

\begin{tabular}{|c|c|c|c|c|c|c|c|c|c|c|}
\hline Espécie vegetais & $\begin{array}{l}\text { Diâmetro } \\
\text { da corola } \\
(\mathrm{mm})\end{array}$ & $\begin{array}{l}\text { Profundidade } \\
\text { da corola } \\
(\mathrm{mm})\end{array}$ & $\begin{array}{l}\text { Cor } \\
\text { da } \\
\text { flor }\end{array}$ & Recurso & $\begin{array}{l}\text { Sexua- } \\
\text { lidade }\end{array}$ & Hábito & Simetria & $\begin{array}{l}\text { Densi- } \\
\text { dade/ha }\end{array}$ & $\begin{array}{l}\text { Tipo de } \\
\text { floração }\end{array}$ & $\begin{array}{l}\text { Número } \\
\text { médio } \\
\text { de flores/ } \\
\text { indivíduo }\end{array}$ \\
\hline Chamaecrista ramosa & 21 & 0 & A & $\mathrm{P}$ & $\mathrm{H}$ & Sar & Z & alta $^{1}$ & St & 3 \\
\hline Humiria balsamifera & 7,8 & 0 & $\mathrm{C}$ & $\mathrm{N}$ & $\mathrm{H}$ & $\mathrm{Ar}$ & $\mathrm{R}$ & 0,55 & St & 40.000 \\
\hline Acosmium bijugum & 12 & 0 & $\mathrm{C}$ & $\mathrm{N} / \mathrm{P}$ & $\mathrm{H}$ & $\mathrm{Ar}$ & $\mathrm{R}$ & 1,11 & St & 30.000 \\
\hline Waltheria cinerescens & 8,6 & 6,8 & A & $\mathrm{N}$ & $\mathrm{H}$ & $\mathrm{Ar}$ & $\mathrm{R}$ & 23,23 & Co & 15 \\
\hline Cuphea brachiata & 13,6 & 9,6 & A & $\mathrm{N}$ & $\mathrm{H}$ & Sar & Z & alta $^{1}$ & St & 20 \\
\hline
\end{tabular}

\section{DISCUSSÃO}

A grande abundância de indivíduos de $X$. cearensis com forte desgaste alar, ao longo de todo o período amostral, sem uma sucessão sazonal, indica que essa espécie tem vida longa e que a presença de indivíduos jovens sugere a ocorrência de multivoltismo.

A distribuição dos ninhos em agregações, como observado para X. cearensis, é comum em abelhas solitárias (WCISLO \& CANE, 1996). Podem ser influenciadas pela disponibilidade de substratos, pela presença de recursos florais e pela preferência dos descendentes em nidificar próximos aos ninhos parentais ou de outros indivíduos coespecíficos (SiHAG, 1993). Em geral, as espécies do gênero Xylocopa não apresentam especialização quanto ao tipo de substrato usado para nidificação (CAMILLO \& GARÓFALO, 1982; CAMILlo et al., 1986). Parece que as características da planta, ao invés da espécie, são os principais fatores de escolha (ANZENBERGER, 1977). Nidificam em uma ampla variedade de madeira morta e/ou apodrecida, compacta e sem rachaduras ou fendas que permitam a penetração de água (HURD \& MouRE, 1963). Especializações a certos tipos de substratos foram observadas entre $X$. sicheli Vachal, 1898 que prefere Aloe sp. (WaTMOUGH, 1974), e X. ciliata Burmeister, 1876 e Eryngium sp. e entre Xylocopa artifex Smith, 1874 em gomos de bambu (SAKAGAMI \& LAROCA, 1971).

A alta freqüência de nidificações em Agaristha revoluta, em Abaeté, parece estar relacionada à grande disponibilidade desta espécie na área que foi a quarta mais densa dentre todas as árvores e arbustos, com maior diâmetro dos galhos em relação às três espécies vegetais mais comuns, cujos galhos não são suficientemente grossos para abrigar ninhos de Xylocopa e a madeira mole, leve e pouco resistente, que favorece a perfuração e formação de galerias. A incidência do número reduzido de ninhos em cada planta ( 1 a 2 ninhos) deve estar relacionada com o diâmetro dos galhos, entre 4 a $17 \mathrm{~cm}$ para a maioria, espaço insuficiente para a construção de outros ninhos sem a conseqüente invasão dos ninhos pré-existentes. $\mathrm{O}$ tamanho das entradas dos ninhos de $X$. cearensis 
apresentou pouca variação. Em geral, em Xylocopa correlaciona-se com o tamanho das espécies que ocupam os ninhos, possibilitando uma melhor defesa das partes internas do mesmo (SAGE, 1968).

Em Abaeté, a principal fonte de pólen para X. cearensis foi Chamaecristha ramosa (Caesalpiniaceae), que apresenta anteras com poros apicais, cujo pólen só pode ser coletado, de modo "legítimo", por vibração. Espécies de outras famílias, como por exemplo Melastomataceae, visitadas por essa abelha, também são poricidas. O uso quase exclusivo de C. ramosa, além de ser uma evidência da especialização dessa abelha ao modo de coleta por vibração, indica também sua preferência por essa espécie vegetal. Enquanto C. ramosa permaneceu florida, as abelhas mantiveram-se constantes, partindo para fontes alternativas apenas nos meses em que o número de flores produzidas por essa planta esteve reduzido.

As abelhas Xylocopa têm importante papel na polinização de plantas tropicais (ENDREss, 1994). VAN DER PIJL (1954) descreveu algumas características de flores com síndrome para polinização por Xylocopa. Dentre elas, destacam-se: floração do tipo "steady-state" (produção diária de pequeno número de flores por longo período); flores grandes, corola com paredes fortes, pálidas ou com cores insaturadas; anteras poricidas; néctar, quando presente, escondido em local protegido da flor. Embora críticas tenham sido feitas a esse tipo de classificação (KUGLER, 1972), por considerar que as mesmas características possam ser associadas a outros grupos de abelhas grandes, que não estão no biótopo, o seu exame pode ajudar a esclarecer alguns aspectos da interação entre flores e Xylocopa. Comparando os critérios propostos porVAN DER PIJL (1954) com as características das plantas predominantemente visitadas por $X$. cearensis, em Abaeté, verifica-se que apenas em $C$. brachiata (fonte de néctar) e $C$. ramosa (fonte de pólen) estes podem ser aplicados. Única ressalva é com relação à cor das pétalas, que em ambas as espécies é amarela saturada. As demais fontes de néctar apresentaram características muito diferentes da classificação de VAN DER PIJL (1954).

Agradecimentos. Ao CADCT/SEPLANTEC-BA, PICDT/CAPES/UFBA e ao CNPq pelo auxílio financeiro; Pe. Jesus Santiago Moure, Universidade Federal do Paraná, pela colaboração na identificação das abelhas; Luciano Paganucci de Queiroz e Ana Maria Giulietti (UEFS) pela identificação das espécies vegetais; aos estagiários do LABEA, pela colaboracão nos trabalhos de campo; ao Destacamento de Proteção ao Vôo do Aeroporto Luis Eduardo Magalhães, Salvador, pelo fornecimento dos dados mensais de precipitação; aos consultores anônimos pelas valiosas críticas e sugestões.

\section{REFERÊNCIAS BIBLIOGRÁFICAS}

Anzenberger, G. 1977. Ethological study of African carpenter bees of the genus Xylocopa (Hymenoptera, Anthophoridae). Z. Tierpsychol., Berlin, 44:337-374.

Buchmann, S. L. 1983. Buzz pollination in angiosperms. In: Jones, C. E. \& LitTle, R. J. eds. Handbook of experimental pollination biology. New York, Van Nostrand Reinhold. p.73-113.

CAmillo, E. \& Garófalo, C. A. 1982. On the bionomics of Xylocopa frontalis (Oliver) in southern Brazil: I - Nest construction and biological cycle. Revta bras. Biol., Rio de Janeiro, 42(3):571-582.

1989. Social organization in reactivated nests of three species of Xylocopa (Hymenoptera, Anthophoridae) in southeastern Brasil. Insectes soc., Paris, 36:92-105.

Camillo, E; Garófalo, C. A. \& Muccillo, G. 1986. On the bionomics of Xylocopa suspecta (Moure) in southern Brazil: nest construction and biological cycle (Hymenoptera: Anthophoridae). Revta bras. Biol., Rio de Janeiro, 46(2):383-393.

Corbet, S. A. \& Willmer, P. G. 1980. Pollination of the yellow passionfruit: nectar, pollen and

Iheringia, Sér. Zool., Porto Alegre, 92(4):47-57, 30 de dezembro 2002 
Carpenter bees. J. agric. Sci. Camb., Cambridge, 95:655-666.

EndRESS, P. K. 1994. Diversity and evolutionary biology of tropical flowers. Cambridge, Cambridge University. 511p.

Frankie, G. W. \& Vinson, S. B. 1977. Scent marking of passion flowers in Texas by females of Xylocopa virginica texana (Hymenoptera: Anthophoridae). J. Kans. ent. Soc., Oklahoma, 50:613-625.

Gerling, D. \& Hermann, H. R. 1978. Biology and mating behavior of Xylocopa virginica L. (Hymenoptera, Anthophoridae). Behav. Ecol. Sociobiol., Heidelberg, 3(2):99-111.

Gerling, D.; Velthuis, W. H. D. \& Hefetz, A. 1989. Bionomics of the large carpenter bee of the genus Xylocopa. Ann. Rev. Entomol., Palo Alto, 34:163-190.

Gottsberger, G.; Camargo, J. M. F. \& Silberbauer-Gottsberger, I. 1988. A bee pollinated tropical community: The beach dune vegetation of Ilha de São Luís, Maranhão, Brazil. Bot. Jb. Syst., Stuttgart, 109(4):469-500.

Greig-Smith, P. 1983. Quantitative plant ecology. Oxford, Blackwell Scientific. 359p.

HuRd, P. D. 1978. An annotated catalog of the carpenter bees (genus Xylocopa Latr.) of the Western Hemisphere (Hymenoptera, Anthophoridae). Washington, Smithsonian Inst. $106 \mathrm{p}$.

Hurd, P. D. \& Moure, J. S. 1963. A classification of the large carpenter bee (Xylocopini). Univ. Calif. Publs Ent., Berkeley, 29:1-365.

Kugler, H. 1972. Zur Bestäubung von Salvia sclarea L. durch Holzbienen (X. violacea L.). Österr. Bot. Z., Wien, 120:77-85.

LAROCA, S. 1995. Ecologia: princípios e métodos. Rio de Janeiro, Vozes. 197p.

Ludwig, J. A. \& Reynolds, J. F. 1988. Statistical ecology: a primer on methods and computing. New York, Wiley. 337p.

Michener, C. D. 1974. The social behavior of the bees; A comparative study. Cambridge, The Belcknap. 404p.

Rebêlo, J. M. \& Garófalo, C. A. 1991. Diversidade e sazonalidade de machos de Euglossini (Hymenoptera, Apidae) e preferências por iscas odores em um fragmento de floresta no sudeste do Brasil. Revta bras. Biol., Rio de Janeiro, 51(4):787-799.

SAGE, R. D. 1968. Observations on feeding, nesting and territorial behaviour of carpenter bees genus Xylocopa in Costa Rica. Ann. ent. Soc. Am., Columbus, 61:884-889.

SaKagami, S. F. \& Laroca, S. 1971. Observations on the bionomics of some Neotropical Xylocopini bees, with some comparative biofaunistic notes (Hymenoptera, Anthophoridae). J. Fac. Sci. Hokkaido Univ., Zool., Sapporo, 18:57-127.

Sakagami, S. F.; Laroca, S. \& Moure, J. S. 1967. Wild bee biocenotics in São José dos Pinhais (Pr), south Brazil. Preliminary report. J. Fac. Sci. Hokkaido Univ., Zool., Sapporo, 19:190-250.

SIHAG, R. C. 1993. Behavior and ecology of the subtropical carpenter bee Xylocopa fenestrata F. J. Apic. Res., Louisiana, 32(2):102-108.

Van Der PiJl, L. 1954. Xylocopa and flowers in the tropics. Proc. K. Ned. Akad. Wet., Amsterdam, 57:413-562.

Watmough, R. H. 1974. Biology and behaviour of carpenter bees in southern Africa. J. Entomol. Soc. Sth. Afr., Hatfield, 37:261-281.

WCIslo, W. T. \& CANE, J. H. 1996. Floral resource utilization by solitary bees (Hymenoptera: Apoidea) and exploitation of their stored foods by natural enemies. A. Rev. Ent., Stanford, 41:257-286

Recebido em 26.07.2002; aceito em 02.09.2002 